\title{
Newborn Transport Practices: Influence on Newborn Survival in Benin City, Nigeria
}

\author{
Ikechukwu Richard Okonkwo ${ }^{1}$, Blessing Imuetinyan Abhulimhen-Iyoha ${ }^{1}$, Angela Anene Okolo ${ }^{1,2, *}$ \\ ${ }^{1}$ Department of Child Health, University of Benin Teaching Hospital, Benin City, Nigeria \\ ${ }^{2}$ Federal Medical Center, Department of Pediatrics, Asaba, Nigeria
}

Email address:

aneneolisa@gmail.com (A. A. Okolo)

${ }^{*}$ Corresponding author

\section{To cite this article:}

Ikechukwu Richard Okonkwo, Blessing Imuetinyan Abhulimhen-Iyoha, Angela Anene Okolo. Newborn Transport Practices: Influence on Newborn Survival in Benin City, Nigeria. American Journal of Pediatrics. Vol. 6, No. 3, 2020, pp. 346-352. doi: 10.11648/j.ajp.20200603.39

Received: July 11, 2020; Accepted: July 27, 2020; Published: August 20, 2020

\begin{abstract}
Objective: To assess the transfer process, modes of transportation, distances covered to access care, condition on arrival, and outcomes of newborns admitted to hospital in Benin City, Nigeria. Methods: This was a cross sectional survey of all consecutively presenting neonates to the children's emergency unit over a 12-month period. Their demographic information, antepartum and perinatal antecedents, clinical information, main complaint, and co-morbidities were noted on a structured questionnaire. Other information gathered included transfer process, mode of transport, distance covered to reach facility, interventions during transport, condition on arrival, and outcome. Data were analyzed using descriptive statistics, frequencies, and chi-square comparisons; significance was set at $\mathrm{P}<0.05$. Results: Responsible persons for 115 babies - 73 males and 42 females_completed the questionnaire. Of the newborns, 62 (53.9\%) were of normal birth weight and $43(37.3 \%)$ of low birth weight; of these $37(32.2 \%)$ were preterm. Most cases arrived from peripheral hospitals without referral notes or prior contact with the receiving hospitals. There was no organized pre- or intra-transfer stabilization or medical interventions. Patients came mainly with relatives $(89 \%)$ or healthcare workers $(10 \%)$. They arrived in private cars $(81 \%)$, and were not kept in warm clothes. Distance covered was $10-20 \mathrm{~km}$ for more than $50 \%$ and over 100 $\mathrm{km}$ for less than $8 \%$. Perinatal asphyxia was the commonest reason for transfer $(14 \%)$. Lower gestational age and cyanosis on presentation significantly affected survival, as only 13 of $25(52 \%)$ with cyanosis and 23 of 37 (62.2\%) preterm babies survived. Overall mortality was 30/ 115 (26.1\%), and preterm mortality was significantly higher at 14/37 (37.8\%). Conclusions: The neonatal transfer process is poorly developed. Prior contact with receiving hospitals is infrequent. Staffing, monitoring, and stabilizing interventions during transfer of sick newborns are inadequate. Transfer mode might have affected survival of preterm infants in particular.
\end{abstract}

Keywords: Neonatology, Newborn Transport, Infant Survival, Nigeria

\section{Introduction}

Neonatal mortality is high worldwide, contributing over $40 \%$ to under-five mortality globally. In Nigeria, neonatal mortality accounts for $32 \%$ of mortality in under-fives and $54 \%$ of infant mortality [1-2]. The major causes of morbidity and mortality include prematurity, birth asphyxia, respiratory tract disorders, and infection. More than $40 \%$ of deliveries occur outside the health system and most deaths occur in community settings. Babies delivered at home or in the community are only taken to health care centers when they develop an illness severe enough to push family members to seek care at primary health care centers (PHC) or private hospitals. These facilities usually lack the resources to cater for sick newborn babies. PHCs are supposed to provide basic obstetric and newborn care, but they are ill equipped to do so. Their limited resources do not support using the bag-mask-valve device for helping babies breathe, a gap alluded to in the "Every Newborn Action 
Plan" launched in 2015 [3]. Health care workers at PHCs often refer sick babies to higher-level health care centers that are mostly located in major cities in Nigeria $[1,2]$. Referrals may be made without contacting the receiving hospitals or sending referral letters. Such notes and especially prior contact could be helpful if these inform and direct immediate and ongoing care. Critically ill babies require safe and timely means of transport to avoid mortality.

Limited access to skilled attendance at birth is still a major challenge [4] in low resource settings (LRS). As a result, infants requiring more intensive supportive care need to be transferred to a center capable of higher-level of neonatal care. Prompt attention to the need for transfer might avert the $50 \%$ of newborn deaths occurring on the first day of life [57]. Many newborn deaths results from lack of contact with the health system or lack of transfer systems to improve access to higher-level facilities [8-11]. In most developing countries, including Nigeria, emergency medical transport systems have yet to be developed. The implementation and expansion of quality new-born care will diminish health care disparities and reduce neonatal mortality. The survival of these babies may hinge on parallel improvement in medical transport mechanisms to access care for the neonate and in health facility quality of care.

In the absence of organized transport, people resort to less safe methods, particularly where babies are delivered outside the health care system or when parents must pay for health care out of pocket. Currently, alongside the basic lack of systems for transportation of sick newborns into the health system in LRS generally, in Nigeria, in particular, there is also a paucity of documentation of what systems do exist.

This report thus documents newborn transport practices and their impact on the survival of babies presenting at the children's emergency room before ultimate transfer to the neonatal unit, with an eye to providing basic data to justify and motivate improvement in these systems.

\section{Participants and Methods}

\subsection{Study site}

This study was conducted in the children's emergency room (CHER) and neonatal unit (SCBU) of the University of Benin Teaching Hospital (UBTH), Benin City: a 550 bed tertiary public health facility in Southern Nigeria. There are no formally established network settings as such. Lowerlevel health facilities in the environs of Benin City refer patients to UBTH. The UBTH is meant to serve the population of Edo state, which has about 4.5 million inhabitants. However, the spread to the other catchment communities' populations of about five thousand inhabitants expands its services to cover the peri-urban and rural areas. The set up for the care of children at the UBTH is representative of what happens nationally in other tertiary health care facilities.

\subsubsection{The CHER}

This unit has bed capacity for twenty-five children, and receives all children who come to the hospital. There is an emergency triage area. The CHER is equipped with a transport incubator for the transfer of newborn babies to the special care baby unit (SCBU), which is located about 50 meters north of this unit, within the delivery suites of the hospital.

The CHER of the UBTH receives an annual admission of 1,631 and a monthly patient load of 136 [10]. These patients' ages range from 0 to 16 years; about 10 to 15 of them are newborns. However, newborn babies are not admitted to this unit; they are only triaged and stabilized here before transfer to the SCBU. The CHER thus serves as the entry point into UBTH for babies.

The CHER is staffed by a team of three consultants with skills in emergency medicine, supported by a team of three senior residents, six junior residents, and four internes.

\subsubsection{The $S C B U$}

This unit receives an average of 80 babies a month, $40 \%$ of whom are babies referred from outside settings. This unit comprises two sections, for in-born and out-born babies, respectively. Each section has a capacity for 25 cots and incubators, separated into three major areas for assigned level of care: high dependency area (HDA), intensive care area (ICA), and an area for the lower risk level care, the SCA.

The unit is run by a complement of six full-time neonatologists, subdivided into three units, and supported by three senior residents and six junior residents. There is also a complement of thirty-seven nurse-midwives with training in pediatric nursing care and some with further neonatal nursing skills. These are the limits of human resources available to support care of such sick babies in Nigeria and most likely in other LRS.

\subsection{Study Population}

All newborn babies presenting to the CHER from February 2015 to January 2016 were included.

\subsubsection{Admission Process and Informed Consent}

Counseling was provided to the families in simple terms and in a language they could understand. During the counseling session, informed verbal parental consent for the use of their child's anonymized data was sought. It was also explained that their refusal of consent for the use of their child's data would not affect their child's treatment. These data were already collected irrespective of this study.

The ethical committee of UBTH approved the protocol for the study and the informed consent procedure.

\subsubsection{Sampling Process}

A survey questionnaire was implemented that addressed the following:

The socio-demographic details, antenatal and intrapartum information, and physical features of babies presenting in the emergency room.

Details of the mode of delivery, birth weight and 
gestational age, place of delivery, APGAR score (where known), mode of transport and details of monitoring or care during transit, accompanying persons, diagnosis before transport, and address of point of transit.

Estimated distance from the point of transport to the facility (determined using Google Earth software through the usual transit roads to the hospital, based on the questionnaire response).

Weight, axillary temperature, and random blood glucose, which were measured on presentation and documented.

The resident doctors in CHER administered this questionnaire. It was piloted and validated among the families who utilized the general practice clinic in another hospital located some $100 \mathrm{~km}$ distant. This enabled its finetuning for use.

\subsubsection{Data Management and Analysis}

The questionnaire was transcribed, entered into spreadsheets, and analyzed using IBM SPSS Statistics 20. In the analysis of data, certain key variables were selected by an a priori hypothesis as modifiers of the outcome. Frequencies and chi-squared tests were utilized for analysis, and statistical significance was set at $\mathrm{p}<0.05$.

\section{Results}

One hundred and fifteen babies were transported to the emergency room within the study period, consisting of 73 males and 42 females.

Table 1, which depicts the sample characteristics, highlights the place and mode of delivery as well as assessment of condition at birth. Home, traditional birth attendant (Table), and maternity ward deliveries together accounted for $17.5 \%$ of all babies in this series. The great majority of babies, however, had been born at a private hospital from where they were transported to the receiving hospital; an additional $2.8 \%$ were born in transit. Many were merely told to take the baby to UBTH without formal letters. Regarding the infants' characteristics and condition at birth, their birth weight ranged from $900 \mathrm{~g}$ to $6000 \mathrm{~g}$; their birth weight categories, gestational age, place of birth, mode of delivery, and APGAR scores were all featured as well. The gestational age range of the babies was between 26 and 42 weeks (Table 1). There were four multiple births in this cohort, consisting of one set of quadruplets and three set of twins. The mean gestational age of 38 weeks was the most common GA, while 42 and 31 weeks were the least.

Table 1. Sample Characteristics.

\begin{tabular}{lll}
\hline Place of birth & $\mathbf{n}(\%)$ & $\mathbf{N}$ \\
\hline Hospital & $90(78.3)$ \\
Home & $9(7.8)$ \\
Traditional birth attendant & $4(3.5)$ \\
Maternity & $8(7.0)$ \\
Transit & $3(2.8)$ & \\
Others & $1(0.9)$ & 115 \\
\hline
\end{tabular}

\begin{tabular}{llll}
\hline Place of birth & $\mathbf{n}(\mathbf{\%})$ & $\mathbf{N}$ & \\
\hline Mode of delivery & & & \\
Spontaneous vertex delivery & $86(74.7)$ & & \\
Breech extraction & $2(1.7)$ & & \\
Elective cesarean delivery & $3(2.6)$ & & \\
Emergency cesarean delivery & $23(20.0)$ & & \\
Assisted vaginal (vacuum) & $1(0.9)$ & 115 & \\
APGAR done & $1^{\text {st }}$ & $5^{\text {th }}$ & \\
Yes & $20(17.4)$ & $18(15.7)$ & $1(0.9)$ \\
No & $95(82.6)$ & $97(84.3)$ & $114(99.1)$ \\
General characteristics & & & \\
Gender & & & \\
Male & $73(63.5)$ & & \\
Female & $42(36.5)$ & 115 \\
Birth weight category & & \\
High birth weight & $4(3.5)$ & \\
Normal birth weight & $62(53.9)$ & \\
Low birth weight & $32(27.8)$ & \\
Very low birth weight & $9(7.8)$ & \\
Extreme low birth weight & $2(1.7)$ & \\
Unknown birth weight & $6(5.2)$ & 115 \\
Gestational age (weeks) & & \\
Term (37-<42) & $78(67.8)$ & \\
Preterm (<37) & $37(32.2)$ & 115 \\
\hline
\end{tabular}

Table 2 depicts the babies' transfer condition.

In the majority of cases, no prior contact was made by referring providers to the receiving hospital; parents were just verbally instructed to go to the receiving health care center, and medical personnel did not accompany patients during transport.

Table 2. Point of Transfer, Distance to Referral Hospital, Mode of Transportation.

\begin{tabular}{lll}
\hline Diagnosis before transport & $\mathbf{n}(\%)$ & $\mathbf{N}$ \\
\hline Yes & $59(51.3)$ & \\
No & $56(48.7)$ & 115 \\
Mode of transport & & \\
Okada (motorbike) & $0(0.0)$ \\
Keke (motor tricycle) & $1(0.9)$ \\
Bus & $16(13.9)$ \\
Car & $93(80.9)$ \\
Ambulance & $4(3.5)$ \\
Foot & $1(0.9)$ \\
Distance to hospital (km) & \\
$\leq 5-10$ & $9(7.9)$ \\
$>10-20$ & $63(54.7)$ \\
$>20-50$ & $17(14.8)$ \\
$>100$ & $9(7.9)$ \\
Untraceable addresses & $17(14.8)$ \\
Point of transport & \\
Home & $40(34.8)$ \\
Hospital & $75(65.2)$ \\
\hline
\end{tabular}

Table 3 depicts characteristics of transportation and events during the transfer process. The majority of cases involved no action taken during the journey; relatives accompanied most of the babies. 
Table 3. Intervention and Availability of Skilled Personnel during Transfer.

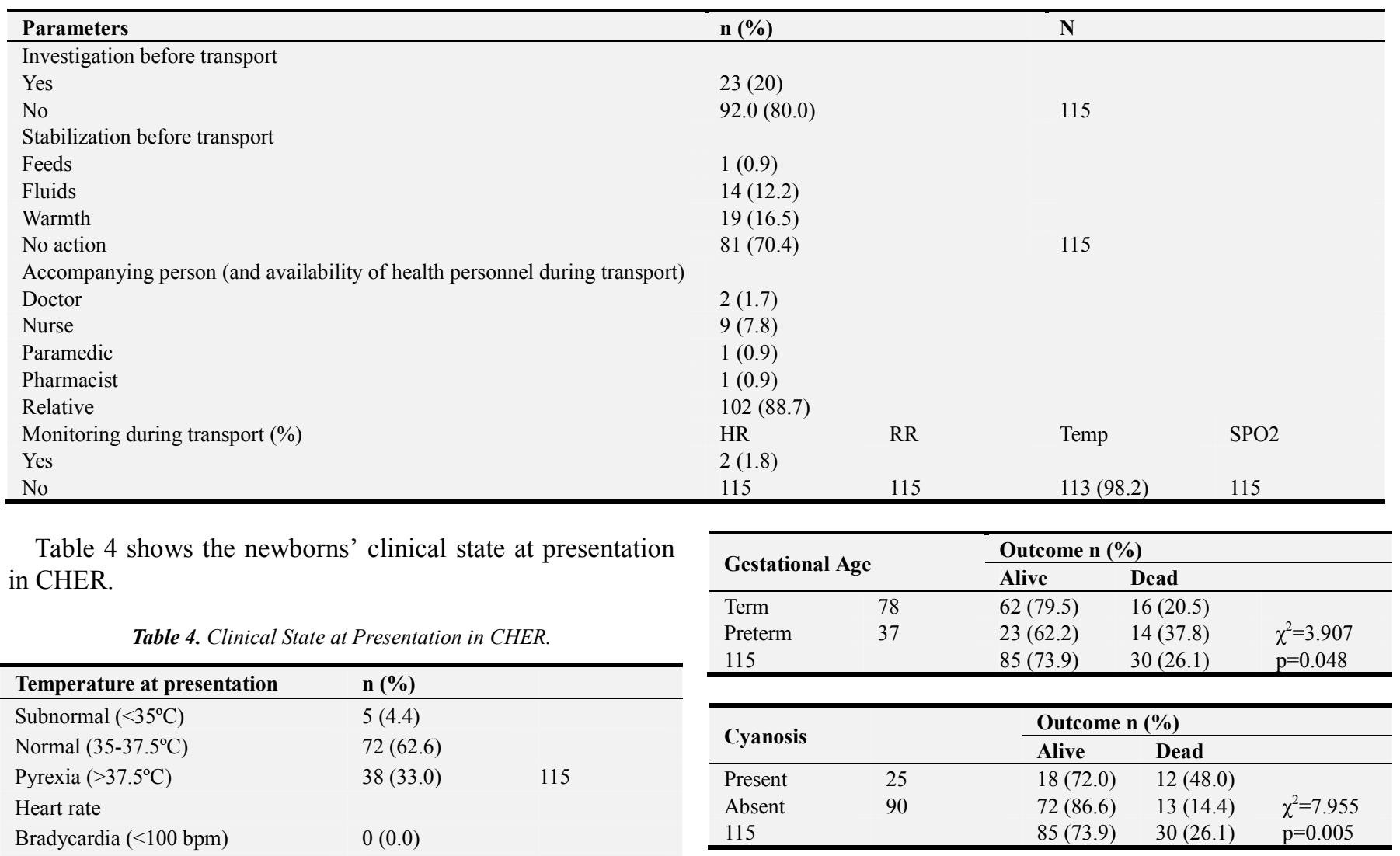

Normal HR (100-160) $99(86.1)$

Tachycardia $(>160)$

$16(13.9)$

Respiratory rate

Gasping

2 (1.8)

Bradypnea (<30 cpm)

2 (1.8)

$65(56.5)$

Tachypnea $(>60 \mathrm{cpm})$

$46(40.0)$

Oxygen saturation

Desaturated $(<90 \%)$

23 (38.3)

Normal saturation $(\geq 90 \%)$

37 (61.7)

60

25 (21.7)

Yes

No

$90(78.3)$

Blood glucose $(\mathrm{mg} / \mathrm{dl})$

Hypoglycemia $(<40)$

Normal (40-150)

5 (7.7)

42 (80.7)

5 (9.6)

Hyperglycemia $(>150)$

Table 5 depicts the outcomes of transferred babies by gestational age and presenting signs. These two variables were selected for assessment of their influence on outcome by an a priori hypothesis. Those born preterm were significantly worse off, as shown by their outcomes.

Table 5. Outcome of Transferred Babies by Gestational Age and Presenting Signs.

\begin{tabular}{ll}
\hline Outcome & n (\%) \\
\hline Alive & $85(73.9)$ \\
Dead & $30(26.1)$ \\
& 115 \\
\hline
\end{tabular}

Figure 1 depicts the kinds of problems for which these babies were brought to hospital.

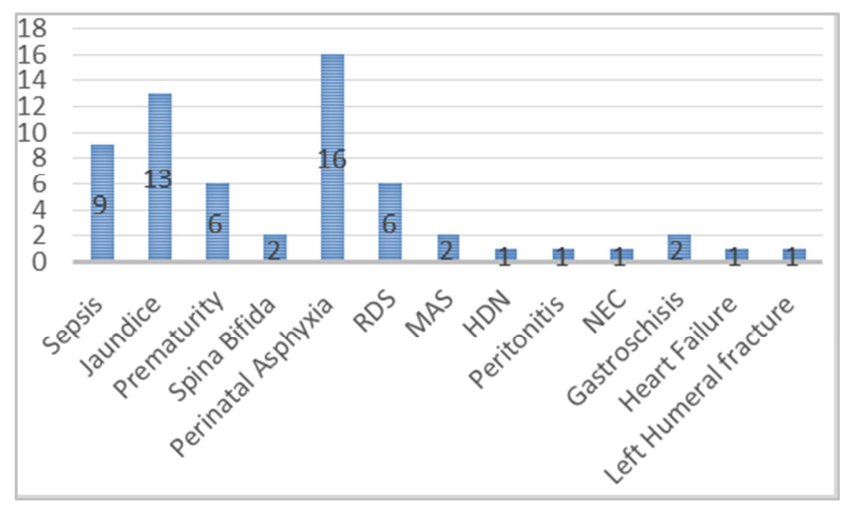

Figure. 1. Spectrum of diagnosis of the transported babies on admission.

\section{Discussion}

The goal of every neonatal transport is to improve the outcome for a critically ill infant who is not near a hospital that provides the required level of intensive care [11]. Safe transfer of newborns is a neglected topic in LRS despite its importance in optimizing new-born care and neonatal survival.

This report shows that the neonatal transport system and infrastructure available in Benin City, southern Nigeria, are far from satisfactory. Males, preterm babies, babies delivered 
in peripheral hospitals, and babies delivered by spontaneous vertex delivery were all more likely to be transported.

Referring hospitals rarely contact receiving hospitals and parents are often left to find their own means for transporting their sick newborns. Long-distance transport of sick infants by car without a healthcare worker in attendance is another suboptimal practice. The finding that $82.5 \%$ of infants were not assessed by APGAR scores at birth, even at 1 minute of life, reflects a low level of neonatal care competency among healthcare workers $[8,13]$.

Although no baby was brought in dead, the eventual outcomes included several deaths, and were affected by gestational maturity and presence of cyanosis at presentation. Simple, low cost, preventive intervention measures might have averted this situation. Given this problematic situation, in utero transfer of women with preterm labor to centers with a higher neonatal care capacity should be emphasized as essential to maximizing the chances of preterm survival. When such transfer cannot be achieved, the Kangaroo Mother Care method should be practiced during the transfer of such babies, who are very often (more than $80 \%$ ) transported in private cars or taxis in the local context $[15,16]$.

None of the babies were transported in transport incubators, nor were they given skin-to-skin care. This reflects a low level of awareness of skin-to-skin care while transporting small infants in our community. This finding differs from that of a survey in Ibadan, by Abdulraheem et al. [17], where up to $31.4 \%$ of transported babies had prolonged skin-to-skin contact during transfer. The degree to which deficiencies in staffing and transport infrastructure contribute to deaths among premature infants needs further study.

Most $(63.5 \%)$ of the transported babies were of male gender; this may be a reflection of the patriarchal nature of our community and preference for male children, but could also be a reflection of the general delivery pattern of ill babies presenting at the time. In the former case, the discrepancy is lower than $84.6 \%$ of referred neonates reported by Harmesh and co-workers in Punjab, India [18]. On the other hand Treleaven and coworkers had severally emphasized the role of preference for sons in Asia where parents were more likely to take sons to hospital more rapidly as more premium is placed on them $[18,19]$. In the context of this survey, the underlying reasons behind the male preponderance amongst the transferred cases is not so clear but may relate to the patriarchal system in this survey's environment where males may be more favored.

More than $75 \%$ of babies were delivered in peripheral hospitals, which, however, did not provide means of transportation for the babies, probably because they had no dedicated means of transportation for referred cases. In most LRS, medical transport and newborn transport is largely seen as the responsibility of parents and caregivers rather than of the healthcare system. The "Essential Care for Every Newborn" [15] training package emphasizes this aspect of care in the skills required for delivery attendants. The propagation of this training package at most health facilities in Nigeria should help rectify this challenge in the Nigerian health system.

Neonatal transport involves effective communication between the referring and receiving hospital, which was not present here, as there was no advance information between the two-centers. Some patients received only verbal instructions as to where to go, with no additional coordination. This is the norm rather than the exception in our local environment.

In this report, only $3.5 \%$ of babies were transported by ambulance, despite their being accompanied by health care personnel, given their critical condition, the level of in-transit monitoring was poor. This also emphasizes the dire need for education on optimal neonatal transport techniques. Such means should ensure the basic needs of the newborn: thermal support, nutrition, respiratory support, resuscitation, and monitoring; however, these cannot be ensured where there has been no prior arrangement with organized transport providers. Some of these basic needs, such as thermal support, but not all of them, may be provided by prolonged skin-to-skin contact with the mother [16-18]. Awareness of the need for these skills and for innovation in service delivery in these areas seems to be lacking. The use of taxis and commercial motorcycles, otherwise referred to as "okada," has been reported $[17,18]$ as a common mode of neonatal transport in Nigeria. In this local environment, their use is banned, and hence none of the cases utilized this means. Motor tricycles, or keke, which are safer, have largely replaced okada in this area but are not used for baby transport, but could be modified for such transport.

The babies were transferred a considerable distance to access care; more than half travelled for $10-20 \mathrm{~km}$, although less than $8 \%$ came from over $100 \mathrm{~km}$ away. Lack of adequate intervention and monitoring while in transport delayed the onset of appropriate supportive interventions, and likely contributed to poor outcomes. This finding reflects the paucity of available neonatal care facilities in the local environment [11].

While a healthcare worker accompanied $12 \%$ of the transported cases, only $2 \%$ of babies had their temperature monitored during transport. For the $88 \%$ transported by relatives, outcomes might be improved by teaching family members to monitor temperature and when feasible utilize skin-to-skin temperature control. Properly targeted strategies for community education and empowerment would improve transport by relatives.

Signs of critical illness were noted on presentation to the Emergency department; including tachypnea (40\%), gasping $(1.8 \%)$, pyrexia $(38 \%)$, cyanosis $(23 \%)$, and desaturation $(38.3 \%)$, and $21.1 \%$ of babies had multiple signs at presentation. Whether these critical signs were present before transport or developed while in transit is not known. Hypothermia, hyperthermia or pyrexia, and hypoglycemia have been shown to result from poor transportation methods and to significantly increase neonatal mortality [17-21].

Cyanosis and prematurity contributed significantly to 
unfavorable outcomes among transferred babies. These danger signs connote adverse neonatal health circumstances, are often noticed in critically ill babies, and have been reported as features of severe illness in babies as well as antecedents of mortality [14, 17, 21].

Perinatal asphyxia (PA) was the commonest reason for neonatal transport in this report. PA might occur in the hospital setting; it is expected to be commoner in settings without a skilled attendant at delivery. The observation in this survey may therefore be a reflection of its prevalence in our communities $[8,9,11]$ where babies are born outside the hospital. Asphyxia is also a major contributor to our national neonatal morbidity and mortality.

The spectrum of diagnosis of the transferred babies reflects the current pattern of morbidities in Nigeria. These conditions are everyday morbidities encountered in most facilities that provide new-born care services. This therefore informs the need for provision of basic care for newborns to cater for such disorders even at lower level health care facilities. Such basic care should provide simple facilities for the early diagnosis and management of these morbidities. It therefore suggests that new strategies for mortality reduction need to focus on providing these basic amenities as a means of more equitable and more widespread access to better quality neonatal care.

The development of an integrated system of care to address newborn transport systems in the context of tertiary newborn care services may be the way forward, linking tertiary care with communities through the Primary Health Care Centers (PHC). Ideally, in developed countries, neonatal transport teams are a component of larger system of perinatal care associated with a tertiary care neonatal intensive care unit [5-7]. The situation is not the same in the study environment or in most LRS. One future direction for the development of effective strategies would lie in establishing such a linkage between the community and tertiary neonatal care providers in our environment. This linkage in the system between community and tertiary care providers was proposed in a recent publication by the Pan American Health Organization [8]. Neonatal transport may be an underappreciated contributor to newborn morbidity and mortality in Nigeria. Health planners need a better understanding of the issue to enable them make-concerted efforts to address this hidden cause of newborn mortality. The Essential Newborn Care (ENCC) was recently launched [15] as training package for frontline health workers and teaches that newborn transport is a desirable intervention to promote. This training will also enhance the capacity of health workers to provide better implementation. As the concept of perinatal care evolves in Nigeria, with the focus on tertiary care facilities, both policy-makers, health planners, and neonatal care practitioners should consider seriously strengthening Maternal Newborn Child Health $(\mathrm{MNCH})$ care services. There is a need to build more effective referral and feedback mechanisms for sick neonates. Such systems need to be integrated into the healthcare delivery system with improved quality and standards of care.

\section{Conclusion and Recommendations}

The neonatal transfer system is poorly developed in Nigeria. The process lacks systematic hospital-to-hospital communication from referral to accepting hospital and feedback to the referring facility. Transfers often occur without appropriate staffing, monitoring, and simple interventions. These deficits in the Nigerian healthcare system must be addressed to improve the quality of medical care and health care outcomes among infants needing higher-level care. Furthermore, medical care generally and newborn transport in particular are poor in Southern Nigeria The poor transport system may be a hidden or at least underappreciated contributor to newborn morbidity and mortality. Health planners need to promote effective implementation and prevention strategies that address this hidden cause of newborn mortality. Essential Newborn Care (ENCC) is one recent intervention that emphasizes the importance of training frontline health workers in newborn transport. Neonatal care practitioners should implement more effective referral and feedback mechanisms to strengthen Maternal Newborn Child Health $(\mathrm{MNCH})$ within the overall healthcare delivery system. In particular, it is crucial to build capacity in neonatal transport as an integrated component of overall perinatal care. ENCC can be a starting point for strengthening health workers' capacity in newborn care. A systematic approach to referrals and feedback could help improve the quality of neonatal transports and could reduce neonatal morbidity and mortality in Nigeria.

\section{Acknowledgements}

The authors wish to recognize and acknowledge the superlative care provided by the resident doctors and the nurses at the children's emergency room upon the arrival of these babies. The authors received no funding for this work, and no competing interests exist.

We would like to thank Editage (www.editage.com) for English language editing.

\section{References}

[1] Akinyemi JO, Bamgboye EA, Ayeni O. Trends in neonatal mortality in Nigeria and effects of bio-demographic and maternal characteristics. BMC Pediatr. 2015; 15: 36.

[2] Austin A, Fapohunda B, Langer A, Orobaton N. Trends in delivery with no one present in Nigeria between 2003 and 2013. Int J Womens Health. 2015; 7: 345-356.

[3] Mary Kinney, Olive Cocoman, Kim E Dickson, Gary L Darmstadt Bernadette Daelmans Nabila Zaka, Natasha Raygaan Rhoda, Sarah Moxon, Lily Kak, Joy E Lawn, Neena Khadka, Gary L Darmstadt. Implementation of the Every Newborn Action Plan: Progress and lessons learned. (2015). Seminars in perinatology 39 (5). DOI: 10.1053/j.semperi.2015.06.004.PubMed.: Every Newborn. 
[4] Moxon SG, Lawn JE, Dickson KE, Simen-Kapeu A, Gupta G, Deorari A, et al. Inpatient care of small and sick newborns: a multi-country analysis of health system bottlenecks and potential solutions. BMC Pregnancy Childbirth. 2015; 15 (Suppl 2): S2-S7.

[5] Karim AM, Admassu K, Schellenberg J, Alemu H, Getachew N, et al. (2013) Effect of Ethiopia's Health Extension Program on Maternal and Newborn Health Care Practices in 101 Rural Districts: A Dose-Response Study. PLoS ONE 8 (6): e65160. doi: 10.1371/journal.pone.0065160.

[6] David Braun, Eric Braun, Vicki Chiu, Anthony E. Burgos, Mandhir Gupta, Marianna Volodarskiy, Darios Getahu. Trends in Neonatal Intensive Care Unit Utilization in a Large Integrated Health Care System. JAMA Network Open. 2020; 3 (6): e205239. doi: 10.1001/jamanetworkopen.2020.5239.

[7] American Academy of Pediatrics, American College of Obstetrics and Gynecology. Guidelines for perinatal care. 7th ed. Elk Grove Village, IL: American Academy of Pediatrics; 2014.

[8] Niermeyer S, Domek G. Neonatal transport in developing country settings: a systematic review. Montevideo: Pan American Health Organization; 2016.

[9] Horbar JD, Badger GJ, Carpenter JH, Kenny MJ, Soll RF, Morrow KA, Buzas JS. Trends in mortality and morbidity for very low birth weight infants, 1991-1999. Pediatrics. 2002; 110 (Part 1): 143-151.

[10] Saving newborn lives in Nigeria: Newborn health in the context of the Integrated Maternal, Newborn and Child Health Strategy. Abuja: Federal Ministry of Health, Save the Children. 2011

[11] Okonkwo I. R, Okolo A. A, Abhulimhen-Iyoha B. Scope of neonatal care services in major Nigerian Hospitals. Niger $\mathrm{J}$ Paediatr. 2016; 43 (1): 8-13.

[12] Abhulimhen-Iyoha BI, Okolo AA. Morbidity and mortality of childhood illnesses at the Emergency Paediatric Unit of the
University of Benin Teaching Hospital, Benin City. Niger J Paediatr. 2012; 39: 71-74.

[13] Lila O'Mahony, George. A. Woodward, in Avery's Diseases of the Newborn (Tenth Edition), 2018 Neonatal Transport.

[14] Ballot, D. E., Chirwa, T., Ramdin, T. et al. Comparison of morbidity and mortality of very low birth weight infants in a Central Hospital in Johannesburg between 2006/2007 and 2013. BMC Pediatr 15, 20 (2015). https://doi.org/10.1186/s12887-015-0337-4.

[15] World Health Organization. Essential newborn care. Geneva: World Health Organization; 1996.

[16] Funk DL, Tilney PVR, Mitchell S, Walker H. Unplanned kangaroo transport of a preterm infant. AMJ. 2012; 6 (31): 264-266. doi: 10.1016/j.amj.2012.08.006.

[17] Abdulraheem MA, Tongo OO, Orimadegun AE, Akinbami FO. Neonatal transport practices in Ibadan, Nigeria. Pan Afr Med J. 2016; 24: 216. doi: 10.11604/pamj.2016.24.216.8651.

[18] Edem M. A. Tette, Benjamin D. Nuertey, Dominic Akaateba and Naa Barnabas Gandau. The Transport and Outcome of Sick Outborn Neonates Admitted to a Regional and District Hospital in the Upper West Region of Ghana: A CrossSectional Study. MDPI Children. Children2020, 7, 22; doi: 10.3390/children 7030022 .

[19] Treleaven E, Toan PN, Le DN, Diamond-Smith N, Partridge JC, Le HT. Gender disparities in child health care seeking in northern Vietnam. Asian Popul Stud. 2016; 12: 312-330.

[20] Treleaven E, Pham TN, Le DN, Brooks TN, Le HT, Partridge JC. Referral patterns, delays, and equity in access to advanced paediatric emergency care in Vietnam. Int $\mathrm{J}$ Equity Health. 2017; 16: 215.

[21] Okawa S, Ansah EK, Nanishi K, Enuameh Y, Shibanuma A, Kikuchi K, et al. High incidence of neonatal danger signs and its implications for postnatal care in Ghana: a cross-sectional study. PLOS One. 2015; 10 (6): e0130712. 